\title{
The Effects of Stacking on the Configurations and Elasticity of Single Stranded Nucleic Acids
}

\author{
A. Buhot and A. Halperin \\ UMR5819 (UJF, CNRS, CEA) DRFMC, CEA Grenoble, \\ 17 rue des Martyrs, 38054 Grenoble cedex 9, France
}

\begin{abstract}
Stacking interactions in single stranded nucleic acids give rise to configurations of an annealed rod-coil multiblock copolymer. Theoretical analysis identifies the resulting signatures for long homopolynucleotides: A non monotonous dependence of size on temperature, corresponding effects on cyclization and a plateau in the extension force law. Explicit numerical results for poly $(\mathrm{dA})$ and poly $(\mathrm{rU})$ are presented.

PACS numbers: 87.15.-v, 61.25.Hq, 87.14.Gg
\end{abstract}

Single stranded nucleic acids (ssNA) experience stacking interactions [1]. These favor parallel orientation of adjacent aromatic rings of the bases giving rise to rigid helical domains. Thus far, the possible coupling of stacking and the elasticity of ssNA received little attention and its existence became recently a subject of debate $[2,3,4]$. The issue is further complicated because the relevant thermodynamic and structural parameters reported vary widely. In this letter we present a theoretical analysis of the configurations and elasticity of ssNA subject to stacking and identify qualitative effects signalling the coupling of stacking with the chains' elasticity. Clear signatures of stacking are discernable in long homopolynucleotides, under high salt conditions when loops do not form and electrostatic interactions are negligible. There are two primary effects. One is the occurrence of a minimum in the radius of the chain, $R$, as the temperature, $T$, is varied. This leads to corresponding effects on the cyclyzation of the chains. The second effect is a plateau in the extension force law of the ssNA subject to tension $f$. The analysis utilizes a model for the helix-coil transition in helicogenic polypeptides [5] modified to allow for the weak cooperativity of stacking. It focuses on the differences between ssNA that stack strongly, polydeoxyadenylate $(\operatorname{poly}(\mathrm{dA}))$ and polyriboadenylate $(\operatorname{poly}(\mathrm{rA}))$, and those that exhibit weak or no stacking, polyribouridylate $(\operatorname{poly}(\mathrm{rU}))$ and polydeoxythymidylate $(\operatorname{poly}(\mathrm{dT}))$. Such long homonucleotides, comprising thousands of nucleotides, can be readily synthesized enzymatically [ $\underline{6}$. The results suggest that under physiological conditions, these effects are important only for poly (dA), poly(rA) and ssNA containing large A domains. In the absence of such domains, stacking effects become noticeable at $T$ lower than $10^{\circ} \mathrm{C}$.

The extension force laws of $\lambda$ ssDNA, as measured in optical tweezers or AFM experiments, do not reveal signatures of stacking 7]. The results can be rationalized by considering ssDNA as freely-jointed chain characterized by a single Kuhn length. This basic picture is augmented to allow for loop formation [8] and for electrostatic interactions [9]. However there is no evidence for large $A$ domains in $\lambda$ ssDNA and these measurements were carried out in ambient $T$ thus precluding, as we shall dis- cuss, significant stacking effects. Stacking effects were reported 2, 3, 4] in "molecular beacons" 10]. These are short ssDNA chains capable of forming stem-loop structures. One end carries a fluorophore and the other a quencher. Accordingly an open hairpin fluoresces and a closed hairpin is quenched. The fluorescence intensity and its fluctuations allow to extract the fraction of hairpins and the opening and closing rate constants. Experiments by the Libchaber group revealed differences in cyclization behavior of poly $(\mathrm{dT})$ and poly $(\mathrm{dA})$ loops that were attributed to stacking and its effects on the rigidity of the chains [2, 3]. This interpretation was disputed by Ansari et al. who ascribed the effects to transient trapping of misfolded loops while arguing that both poly $(\mathrm{dT})$ and poly $(\mathrm{dA})$ behave as flexible polymers [4]. Our analysis does not pertain directly to this system since it concerns long chains. However, the resulting predictions identify clear signatures of stacking when misfolding is not an option.

Stacking involves interactions between nearest neighbors and is thus non-cooperative or weakly cooperative [1]. It involves a broad transition between the stacked, helical state obtained at low $T$, and randomcoil configurations at high $T$. At intermediate $T$, ssNAs comprises of stacked domains interspaced with "melted", random-coil ones. The polydispersed domains undergo dynamic equilibrium and the overall behavior is of annealed rod-coil multiblock copolymer. The strength of the stacking interactions vary with the identity of the bases. It is strongest between adenosines (A) and it is weakest among uridines (U) and thymines ( $\mathrm{T}$ ). The interactions between chemically different bases are weak. Thus, stacking is most pronounced in poly $(\mathrm{dA})$ and poly $(\mathrm{rA})$ while it is weak in poly $(\mathrm{rU})$ and in heteropolynucleotides without extended A domains. There is evidence that stacking does not occur in poly $(\mathrm{dT})$. In every case, stacking is insensitive to the concentration of salt. We consider ssNA homoplymers comprising $N \gg 1$ identical monomers, nucleotides, of which $\theta N$ are stacked. The stacked bases form $y N$ helical domains. In comparison to the non-stacked bases, the excess free energy of each stacked monomer is $\Delta f=\Delta h-T \Delta s . \Delta h$ reflects the enthalpy gain associated with the stacking 
while $\Delta s$ allows for the loss of configurational entropy due to the parallel orientation of the stacked bases. The reported values of $\Delta h$ and $\Delta s$ vary widely. For poly(rA) $\Delta h$ ranges between -3 to $-10 \mathrm{kcal} /$ mole while $\Delta s$ values span the range -10 to -27 e.u.. In our calculations we will use two sets of values: $\Delta h=-13 \mathrm{kcal} / \mathrm{mole}$ and $\Delta s=-40$ e.u. as reported for poly $(\mathrm{dA})$ as well as $\Delta h=-2.7 \mathrm{kcal} / \mathrm{mole}$ and $\Delta s=-10$ e.u. as reported for U stacks [1]. This choice brackets the range of reported parameters and will allow us to set tentative boundaries of the experimental conditions to explore. The terminal monomers of the domain experience stacking interaction with one neighbor rather than two. The reduction in their configurational entropy is possibly weaker. To allow for these two effects we assign each terminal monomer with an additional free energy $\Delta f_{t}$. The corresponding Zimm and Bragg parameters are $s=\exp (-\Delta f / k T)$ and $\sigma=\exp \left(-2 \Delta f_{t} / k T\right)$ [1]. The $\theta$ vs $T$ melting curves are broad leading to $0.5 \leq \sigma \leq 1$ and suggesting weak cooperativity. For simplicity we will assume perfect non-cooperative behavior with $\sigma=1$. For comparison, in helicogenic polypeptides the $i$ th monomer binds the monomer $i+3$ thus giving rise to higher cooperativity signalled by much smaller $\sigma$ values, of order $10^{-2}-10^{-3}[12$. The distance between the bases in the stacked form varies between $0.32 \mathrm{~nm}$ and $0.35 \mathrm{~nm}$ depending on the measurement technique and $T$. In the following we will thus assign a value of $b=0.34 \mathrm{~nm}$ to the projected length of a stacked monomer along the axis of the helical domain. Because of the non-cooperativity of the stacking the helical domains are relatively short. While their persistence length is not known we will assume that it is much longer than the typical domain length and thus effectively infinite. In contrast we assume that the unstacked domains behave as freely jointed chains and we neglect excluded volume effects. Two parameters thus characterize the coil-like domains: the effective length of an unstacked monomer, $a$, and the persistence length of the coil, $\lambda$. Neither is well established. A common value for $a$ is $0.6 \mathrm{~nm}$ [7] while reported values of $\lambda$, for stackingfree chains vary between $0.75 \mathrm{~nm}$ and $3.5 \mathrm{~nm}$ [13]. We will utilize $\lambda=a$ and $\lambda=3.5 \mathrm{~nm}$. The free energy of an unconstrained homonucleotide within this model is [5]:

$$
\begin{aligned}
\frac{F_{0}}{N k T} & =-\theta \ln s+y \sum_{n}\left[p_{s}(n) \ln p_{s}(n)+p_{u}(n) \ln p_{u}(n)\right] \\
& -\sum_{n}\left[\left(\mu_{1}^{s}+n \mu_{2}^{s}\right) p_{s}(n)+\left(\mu_{1}^{u}+n \mu_{2}^{u}\right) p_{u}(n)\right] . \quad(1)
\end{aligned}
$$

The first term allows for the excess free energy of the stacked bases. The next two terms specify the mixing entropy arising from the polydispersities of the stacked and coil-like domains where $p_{s(u)}(n)$ is the probability of stacked (unstacked) domain comprising of $n$ bases. The last four terms impose two constraints by use of Lagrange multipliers. $\mu_{1}^{s(u)}$ assures the probability normalization while $\mu_{2}^{s(u)}$ imposes the average number of monomers, $\theta / y$ and $(1-\theta) / y$ respectively, in these domains [5]. The

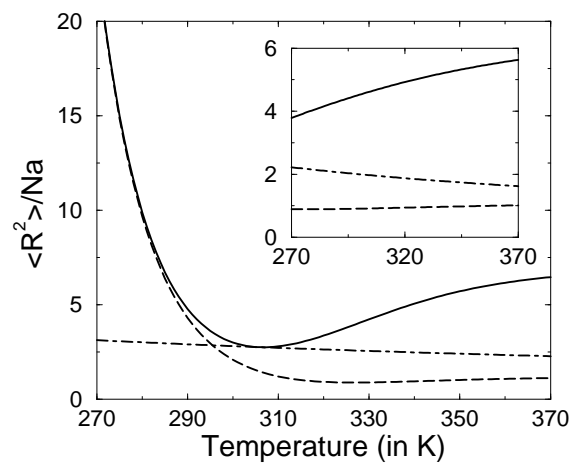

FIG. 1: Plots of $\left\langle R^{2}\right\rangle / N a=2 l_{p}$ vs. $T$ for poly(dA) with $l_{p}$ given by Eq. (3) exhibit a minimum at $T_{\min }=307 \mathrm{~K}$ for $\lambda=3.5 \mathrm{~nm}$ (full line), $T_{\min }=327 \mathrm{~K}$ for $\lambda=0.6 \mathrm{~nm}$ (dashed line) in comparison with $\left\langle R^{2}\right\rangle / N a$ vs. $T$ when $l_{p}=\kappa / k T$ (dotdashed line). $\kappa$ was set by equating $l_{p}$ at $T_{\min }$ for $\lambda=3.5 \mathrm{~nm}$. The inset depicts the same plots for poly $(\mathrm{rU})$.

equilibrium conditions, $\partial F_{0} / \partial \theta=\partial F_{0} / \partial y=0$, yield $\theta=$ $s /(s+1), y=s /(s+1)^{2}=\theta(1-\theta), p_{s}(n)=(1-\theta) \theta^{n-1}$ and $p_{u}(n)=\theta(1-\theta)^{n-1}$. The average number of bases in a stacked and unstacked domain are thus respectively $\langle n\rangle_{s}=\sum_{n} n p_{s}(n)=1 /(1-\theta)$ and $\langle n\rangle_{u}=\sum_{n} n p_{u}(n)=$ $1 / \theta$.

The simplest characteristic of stacking is the $T$ dependence of the mean square radius of the chain, $\left\langle R^{2}\right\rangle$, as determined by the end-to-end distance $R$. Assuming that the rod and coil segments are freely jointed, the contributions of stacked and unstacked monomers to $\left\langle R^{2}\right\rangle$ are independent. The $(1-\theta) N$ unstacked monomers in coil domains with a persistence length $\lambda$ constitute $N a(1-\theta) / 2 \lambda$ freely jointed segments of length $2 \lambda$ contributing $(2 \lambda)^{2} N a(1-\theta) / 2 \lambda$ to $\left\langle R^{2}\right\rangle$. Of the $\theta N$ stacked monomers, the ones that form domains incorporating $n$ bases contribute $N \theta p_{s}(n) /\langle n\rangle_{s}$ freely jointed segments of length $n b$. Altogether,

$$
\left\langle R^{2}\right\rangle=N 2 \lambda a(1-\theta)+N b^{2} \theta\left\langle n^{2}\right\rangle_{s} /\langle n\rangle_{s}
$$

where $\left\langle n^{2}\right\rangle_{s} /\langle n\rangle_{s}=(1+\theta) /(1-\theta)$ [14]. The qualitatively important feature of $\left\langle R^{2}\right\rangle$ is a minimum (Fig.1) at $\theta_{\text {min }}=1-\sqrt{2 b^{2} /\left(2 \lambda a+b^{2}\right)}$. This effect disappears if we ignore the differences between the size of the monomer in the two states $\left(2 \lambda a=b^{2}\right)$. It reflects a competition between two contributing processes: (i) the number of effective monomers increases with $T$ because the number and the size of the helical domains decrease, and (ii) the size of the chain at low $T$ is dominated by a single helical domains whose length scales with $N$ rather than with $N^{1 / 2}$. For the values of $a$ and $b$ we utilize the minimum is attained at $\theta=0.769$ or $s=3.32$ for $\lambda=3.5 \mathrm{~nm}$ and at $\theta=0.474$ or $s=0.9$ for $\lambda=0.6 \mathrm{~nm}$. Thus for poly $(\mathrm{dA})$ the corresponding $T_{\min }$ values are $T_{\min }=33.6^{\circ} \mathrm{C}$ and $T_{\min }=53.5^{\circ} \mathrm{C}$ while for poly $(\mathrm{rU}) T_{\min }=-55^{\circ} \mathrm{C}$ and $T_{\min }=2.5^{\circ} \mathrm{C}$. A closely related effect occurs in helicogenic polypeptides as they undergo a cooperative helixcoil transition [12]. 
ssNA chains are occasionally considered as semiflexible, worm-like chains. In this case, it is implicitly assumed that the properties of the chain along its backbone are uniform. In the limit of $N \gg 1$ semiflexible chains obey $\left\langle R^{2}\right\rangle=2 l_{p} L$ where $l_{p}$ is the persistence length and $L=N a$ is the contour length. Within this model $l_{p}=\kappa / k T$ where $\kappa=$ const is the bending modulus of the chain. If the behavior of the ssDNA is analyzed within this framework while the chain obeys the stacking model, the $T$ dependence of $l_{p}$ thus extracted is given by

$$
2 l_{p}=2 \lambda(1-\theta)+\left(b^{2} / a\right) \theta(1+\theta) /(1-\theta) .
$$

At high $T$, when $\theta \rightarrow 0, l_{p} \approx \lambda$ while for low $T$, when $\theta \rightarrow$ 1 it diverges as $l_{p} \sim b^{2} / a(1-\theta) \sim b^{2} \exp (-\Delta f / k T) / a$. This $T$ dependence is markedly different from the $1 / T$ behavior predicted by the worm-like chain model (Fig.1).

A related signature involves cyclization reactions. $\mathrm{Cy}-$ clization reactions require the two ends of the chain to be within a certain capture radius, $r_{c} \ll\left\langle R^{2}\right\rangle^{1 / 2}$. The thermodynamics of the ring formation are determined by $P(R) d R$, the probability for the end-toend distance of the chain to be in the range $R$ to $R+d R$. When $N \gg 1, P(R)$ of flexible homopolymers, behaving as freely jointed chains with constant monomer size, assumes a Gaussian form 15]: $P(R)=$ $4 \pi R^{2}\left(2 \pi\left\langle R^{2}(\theta)\right\rangle / 3\right)^{-3 / 2} \exp \left(-3 R^{2} / 2\left\langle R^{2}(\theta)\right\rangle\right)$. This result applies to ssDNA in the limit of $\theta \rightarrow 0$, when the effect of the stacking is negligible. Clearly, it is wrong when $\theta \rightarrow 1$ and the configurations are dominated by a single, long stacked domain. For $\theta>0$, the Gaussian form is valid provided $N \gg 1$ and $y N \gg 1$ i.e., the stacked monomers form a large number of domains. The polymer may then be considered as a freely-jointed chain whose effective monomers are rod-coil diblocks of varying sizes. The Gaussian form is applicable in this regime since the probability distribution of lengths of the rod-coil "monomers", while unspecified, is identical for all rod-coil diblocks [16]. The cylization equilibrium is ruled by the elastic free energy arising from constraining $R, F_{e l}=-k T \ln P(R)=-k T \ln \left(R^{2} /\left\langle R^{2}(\theta)\right\rangle^{3 / 2}\right)+$ $3 k T R^{2} / 2\left\langle R^{2}(\theta)\right\rangle+$ cste. This $F_{e l}$ has negligible effect on $\theta$ and $y$ because its contribution to the equilibrium conditions arises from $R^{2} /\left\langle R^{2}(\theta)\right\rangle$. For $R \ll N^{1 / 2} a$ the corresponding terms scale as $1 / N$ and are thus negligible. To obtain the precise cyclization penalty it is important to allow for the weighted contributions of all the configurations with $R \leq r_{c}$. Since for $r_{c} \ll\left\langle R^{2}(\theta)\right\rangle^{1 / 2}$ the exponent in $P(R)$ is of order unity, the fraction of cylizable states within the freely jointed chain model is $\int_{0}^{r_{c}} P(R) d R \sim\left[r_{c} /\left\langle R^{2}(\theta)\right\rangle^{1 / 2}\right]^{3}$. For self avoiding chains $\left\langle R^{2}(\theta)\right\rangle^{1 / 2}$ is replaced by the $\theta$ dependent Flory radius [17]. Altogether the cyclization entropy for $r_{c} \ll$ $N^{1 / 2} a$ is $S_{c y c}(\theta)=3 k \ln \left[r_{c} /\left\langle R^{2}(\theta)\right\rangle^{1 / 2}\right]$ and the equilibrium constant for the cyclization reaction is specified by $k T \ln K_{c y c}=\Delta H-T S_{c y c}(\theta)$ where $\Delta H$ is the binding enthalpy of the terminal groups. The activation free energy for cyclization, $\Delta F_{c y c}^{\ddagger}$ may be identified with $-T S_{c y c}(\theta) \sim k T \ln \left\langle R^{2}(\theta)\right\rangle^{3 / 2}[3]$. This $\Delta F_{c y c}^{\ddagger}$ exhibits a minimum at $\theta_{\min }$ and thus at the corresponding $T_{\min }$. We should emphasize that this analysis is not valid when the chain contains a large stacked domain comprising most of the monomers. In this case the bending of the chain can induce melting of the stacked domain thus introducing a coupling of $\theta$ and the cyclization: The elastic free energy of fully stacked chain of length $L=l_{p}=\kappa / k T$ forming a ring of radius $R=L / 2 \pi$ is $\kappa L / 2 R^{2}=2 \pi^{2} k T \approx 20 k T$ while the reported stacking free energy per base at $25^{\circ} \mathrm{C}$ is in the range of 2 to $8 k T$. This rough argument suggests that the chain can lower its free energy by "melting" a few stacks thus avoiding the bending penalty.

When the reaction between the terminal groups is diffusion controlled the cyclization rate constant assumes the form $k_{\text {cyc }} \sim 1 / \tau$ where $\tau$ is the longest characteristic time of the chain [18. If hydrodynamic interactions are neglected $\tau=\tau_{R}$ where $\tau_{R} \approx\left(\eta_{s} a^{3} / k T\right) N^{2}$ is the Rouse time and $\eta_{s}$ is the solvent viscosity. Allowing for hydrodynamic interaction leads to $\tau=\tau_{Z}$ where $\tau_{Z} \approx \eta_{s} R^{3} / k T$ is the Zimm time. When excluded volume interaction are negligible $\tau_{Z} \sim N^{3 / 2}$ while in the opposite case $\tau_{Z} \sim N^{3 \nu}$ where $\nu=0.588$. Experimental studies of cyclization of synthetic polymers in non-aqueous solutions are consistent with $k_{c y c} \sim N^{-3 / 2}$. Our discussion suggests thus that the cyclization rate constant of long ssDNA will follow $k_{c y c} \sim\left\langle R^{2}(\theta)\right\rangle^{-3 / 2}$ with a maximum at $\theta_{\min }$.

The $T$ dependence of $R, K_{c y c}$ and $k_{c y c}$ is recovered if we consider the ssDNA as a worm like chain with a presistence length $l_{p}$ given by (3). This picture fails qualitatively when considering the extension force law. To obtain it, we augment $F_{0}$ with the elastic free energy, $F_{e l}$, of a freely-jointed chain subject to tension $f[19]$. A Kuhn length $2 \lambda$ is assigned to the coil domains while each of the rigid helical domains orients as an independent effective monomer. Allowing for the size distribution of the stacked, helical domains we obtain [5]

$$
\frac{F_{e l}}{N k T}=-\frac{1-\theta}{\delta} \mathcal{L}_{i n t}(\delta x)-y \sum_{n} p_{s}(n) \mathcal{L}_{i n t}(\gamma n x)
$$

where $\delta=2 \lambda / a, \gamma=b / a, \mathcal{L}_{\text {int }}(x)=\ln [\sinh (x) / x]$ and $x=f a / k T$. Minimization of $F=F_{0}+F_{e l}$ in the constant $f$ ensemble yields $\theta, y, p_{s(u)}(n)$ [20] and the force law in the form

$$
\frac{R}{N a}=(1-\theta) \mathcal{L}(\delta x)+\theta \gamma \operatorname{coth}(\gamma x)-\frac{\theta \gamma s A}{\sinh (\gamma x)}-\frac{y}{x} .
$$

At low $T$ the force laws obtained from this model exhibit a smoothed plateau associated with the enhancement of stacking by the applied force (Fig.2). Initially, the extension lowers the entropy of the chain thus favoring the ordered, stacked domains. Eventually, further stretching enforces melting of the stacks in order to release the stored length $(b=0.34 \mathrm{~nm}$ vs $a=0.6 \mathrm{~nm})$. For our choice of parameters, poly $(\mathrm{dA})$ exhibits a pronounced plateau at $T=25^{\circ} \mathrm{C}$ while for poly $(\mathrm{rU})$ significant deviations from the freely jointed chain appear below $T=10^{\circ} \mathrm{C}$. No deviations are expected for poly $(\mathrm{dT})$. Modelling the chain 


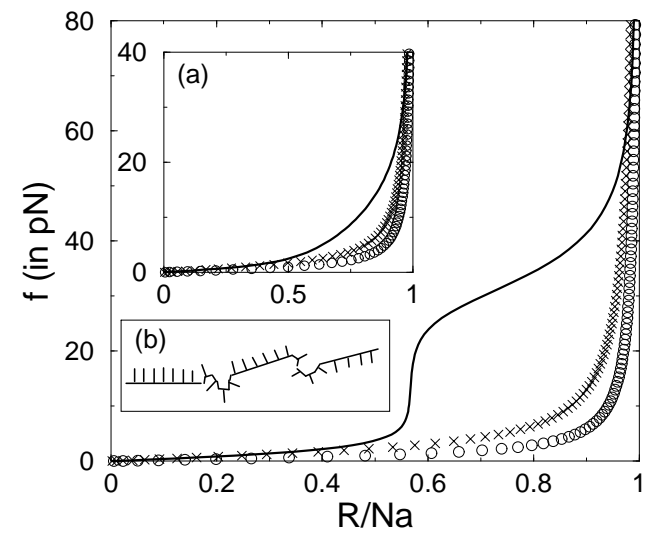

FIG. 2: Plots of $f$ vs $R / N a$ for poly $(\mathrm{dA})$ at $T=25^{\circ} \mathrm{C}$ as obtained from the stacking model Eq. (5) with $\lambda=3.5 \mathrm{~nm}(-$ $-)$, from the freely jointed chain model with $l_{p}$ given by Eq. (3) $(\mathrm{xxxx})$ and with $l_{p}=3.5 \mathrm{~nm}$ (oooo). Insets: (a) depicts the same plots for poly $(\mathrm{rU})$ at $10^{\circ} \mathrm{C}$. (b) Schematic picture of ssDNA with stacked blocks.

as a freely jointed chain with a $T$ dependent Kuhn length (3) does not recover this plateau because it does not allow for the coupling between the stacking and the extension. Accordingly, single molecule stretching experiments provide a stringent test allowing to distinguish between the conflicting views on the effect of stacking on the elasticity of ssDNA.

Straightforward modification of models for the helixcoil transition in polypeptides allowed us to study the effects of stacking interactions on the configurational and elastic properties of homonucleotides. In particular, we investigated the dependence of $\left\langle R^{2}\right\rangle^{1 / 2}, K_{c y c}, k_{c y c}$ and the force laws, $f$ vs $R$, on $T$. The most noticeable effects are extrema in the plots of $\left\langle R^{2}\right\rangle^{1 / 2}$ and $k_{c y c}$ vs. $T$ as well as the appearance of a plateau in the force law. The numerical results are based on reported values of $\Delta h, \Delta s$, $a, b$ and $\lambda$ as obtained from experiments. In confronting our predictions with future experiments it is important to note that the reported parameters vary widely with the measurement technique and the experimental conditions. They also depend on the model used to analyze the data. With this caveat in mind, the qualitative effects we discuss provide powerful diagnostics for the coupling of stacking interactions with the elastic properties of ssNA. These predictions are meaningful because spectroscopic evidence indicates significant stacking in poly $(\mathrm{dA})$ and poly $(\mathrm{rA})$ at $20^{\circ} \mathrm{C}$, irrespective of the precise values of $\Delta h, \Delta s, a$ and $\lambda$. The results presented above can also be used to test the performance of various sets of $\Delta h$, $\Delta s, a, b$ and $\lambda$ in recovering the observable $\left\langle R^{2}\right\rangle^{1 / 2}$ vs $T$ or $f$ vs $R$ plots.
[1] CR Cantor and PR Schimmel, Biophysical Chemistry (Freeman, New York, 1980); VA Bloomfield, DM Crothers and I Tinoco, Nucleic Acids: Structure, Properties and Functions (University Science Books, Sausalito, USA, 2000).

[2] NL Goddard et al., Phys. Rev. Lett. 85, 2400 (2000); Phys. Rev. Lett. 88, 069802 (2002).

[3] DP Aalberts, JM Parman and NL Goddard, Biophys. J. 84, 3212 (2003).

[4] A Ansari, SV Kuznetsov and Y Shen, Proc. Natl. Acad. Sci. USA 987771 (2001); SV Kuznetsov et al., Biophys. J. 81, 2864 (2001); Y Shen, SV Kuznetsov and A Ansari, J. Phys. Chem. B 105, 12202 (2001); A Ansari, Y Shen and SV Kuznetsov, Phys. Rev. Lett. 88, 069801 (2002).

[5] A Buhot and A Halperin, Phys. Rev. Lett. 84, 2160 (2000); Macromolecules 35, 3238 (2002).

[6] FJ Bollum in The Enzymes Vol. X, PD Boyer ed. (Academic Press, New York, 1974); T Godefroy-Colburn and M Grunberg-Manago in The Enzymes Vol. VII, PD Boyer ed. (Academic Press, New York, 1972).

[7] S Smith, Y Cui and C Bustamante, Science 271, 795 (1996); TR Strick et al., Rep. Prog. Phys. 66, 1 (2003).

[8] A Montanari and M Mezard, Phys. Rev. Lett. 86, 2178 (2001).

[9] Y Zhang, H Zhou and ZC Ou-Yang, Biophys. J. 81, 1133 (2001); MN Dessinges et al., Phys. Rev. Lett. 89, 248102 (2002).

[10] S Tyagi and FR Kramer, Nature Biotech. 14, 303 (1996).

[11] BH Zimm and IK Bragg, J. Chem. Phys. 31526 (1959).

[12] TM Birshtein and OB Ptitsyn, Conformation of Macro- molecules (John Wiley, New York, 1966).

[13] JB Mills, E Vacano and PJ Hagerman, J. Mol. Biol. 285, 245 (1999).

[14] $\left\langle R^{2}\right\rangle$ for small $\theta$ can be approximated by ignoring polydispersity of the helical domains. The ssNA comprises thus of $N a(1-\theta) / 2 \lambda$ unstacked domains of size $2 \lambda$ and $N \theta(1-\theta)$ helical domains of length $N b /(1-\theta)$. Both the unstacked and helical domains are freely jointed leading accordingly to $\left\langle R^{2}\right\rangle \simeq N 2 \lambda a(1-\theta)+N b^{2} \theta /(1-\theta)$.

[15] M Doi and SF Edwards, The Theory of Polymer Dynamics (Oxford Univerity Press, 1986).

[16] S Chandrasekhar, Rev. Mod. Phys. 15, 1 (1943).

[17] PG de Gennes, Scaling Concepts in Polymer Physics (Cornell University Press, 1979).

[18] C Cuniberti and A Perico, Prog. Polym. Sci. 10, 271 (1984).

[19] For a chain consisting of $N$ freely jointed monomers of length $a$, each monomer is assigned an energy of $-f a \cos \phi$ where $\phi$ is the angle between the axis of the segment and the direction of $f$. The end-to-end distance satisfies $R / N a=\mathcal{L}(x)$ where $\mathcal{L}(x)=\operatorname{coth}(x)-1 / x$ is the Langevin function and $x=f a / k T$. The elastic free energy in the constant $f$ ensemble is $-F_{\text {el }}=\int_{0}^{f} R\left(f^{\prime}\right) d f^{\prime}=$ $N k T \mathcal{L}_{\text {int }}(x)=N k T \ln [\sinh (x) / x]$.

[20] $\theta$ and $y$ are solutions of $\theta \gamma x\left(1-s A e^{\gamma x}\right)\left(1-s A e^{-\gamma x}\right)=$ $(1-\theta-y) s A(x, \theta, y) \sinh (\gamma x)$ and $2 \gamma x y=(1-\theta-y) \ln [(1-$ $\left.\left.s A e^{-\gamma x}\right) /\left(1-s A e^{\gamma x}\right)\right]$ where $A(x, \theta, y)=[(1-\theta-y) /(1-$ $\theta)][\delta x / \sinh (\delta x)]^{1 / \delta}$. The probabilities $p_{s(u)}(n)$ are specified by $p_{s}(n)=(1-\theta-y)(s A)^{n} \sinh (n \gamma x) / n \gamma x y$ and $p_{u}(n)=[(1-\theta-y) /(1-\theta)]^{n} y /(1-\theta-y)$. 